\title{
Pouvoir et prière dans les images byzantines de don
}

\section{Tania Kambourova}

\section{Q OpenEdition}

Journals

Édition électronique

URL : https://journals.openedition.org/cem/5612

DOI : $10.4000 /$ cem.5612

ISSN : 1954-3093

Éditeur

Centre d'études médiévales Saint-Germain d'Auxerre

\section{Référence électronique}

Tania Kambourova, "Pouvoir et prière dans les images byzantines de don », Bulletin du centre d'études médiévales d'Auxerre / BUCEMA [En ligne], Histoire de l'art \& Anthropologie, mis en ligne le 29 août 2008, consulté le 24 septembre 2022. URL : http://journals.openedition.org/cem/5612 ; DOI : https://doi.org/ $10.4000 /$ cem. 5612

Ce document a été généré automatiquement le 24 septembre 2022.

\section{c) (†) (2)}

Creative Commons - Attribution - Pas d'Utilisation Commerciale - Partage dans les Mêmes Conditions 4.0 International - CC BY-NC-SA 4.0

https://creativecommons.org/licenses/by-nc-sa/4.0/ 


\title{
Pouvoir et prière dans les images byzantines de don ${ }^{1}$
}

\author{
Tania Kambourova
}

\section{Cadre conceptuel et méthodologique}

1 L'un des points de rencontre possibles entre l'anthropologie et l'histoire de l'art est sans doute l'approche interdisciplinaire que met en place l'anthropologie historique lorsqu'elle s'intéresse aux images. En effet, pour faire un bref rappel, ce courant historiographique apparaît en France en réaction à l'histoire positiviste du XIXe siècle, et en rapport avec le développement général des sciences sociales, autour de la Revue des Annales, en 1929. Pourtant à ses débuts, l'anthropologie historique, qui s'intéressait à des problématiques socio-anthropologiques comme la question de la parenté, des mythes, de la mort, de la mémoire, du corps, du don, etc., délaissait au second plan les images en tant que documents figurés d'une époque. Cet intérêt prononcé pour les images s'affirme avec la génération d'historiens comme G. Duby, J. Le Goff, J.-Cl. Schmitt, J. Baschet ${ }^{2}$. Ces dernières décennies on assiste à la mise en place d'une véritable anthropologie visuelle, à sa théorisation et à l'élaboration de sa propre méthodologie pour l'analyse des images médiévales. Plusieurs points me semblent importants à souligner.

1. Les images sont traitées dans leur spécificité propre, et même lorsqu'elles se rapportent étroitement à un texte, elles ne sont pas considérées comme son illustration, car les modes figuratif et verbal fonctionnent différemment notamment sur le plan de la temporalité (irruption figurative totale, simultanée sur la surface vs linéarité et succession des caractères).

2. Les images possèdent une autonomie sémantique, mais ne sont pas isolables de la série où elles sont inscrites dans l'iconographie ou dans un contexte historique plus large. Aussi leur ancrage dans l'ensemble de l'imaginaire social dévoile-t-il les enjeux de pouvoir et de mémoire qui leur sont constitutifs. Il n'y a pas une réalité qui se cache derrière l'image, car tout le réel est déjà dans l'image.

3. Sans leur dénier leur valeur esthétique, les images ne possèdent pas une autonomie fonctionnelle, à la manière dont commencent à être perçues les œuvres d'art en Flandre et en Italie au XVe siècle ( $C^{\prime}$ est sur ce point que divergent les opinions de deux anthropologues 
et historiens - J.-Cl. Schmitt et H. Belting. En effet pour ce dernier seule la fonction cultuelle est prédominante dans les images médiévales, alors que le premier distingue une gamme de formes "cultuelles" différentes et souligne la valeur esthétique de celles-ci).

4. Une des notions clé pour comprendre les images médiévales est celle d'imago qui recouvre à la fois leur aspect matériel (images verbales et figuratives), mental (images mentales, rêves, etc..) et anthropologique (l'homme est fait à l'image de Dieu comme il est annoncé dans la Genèse).

5. Ce sont quelques-uns des critères différenciant les images médiévales des images modernes et qui sous-tendent le travail spécifique avec les images en anthropologie historique.

2. Image et icône, imago et eikôn

2 Une fois la spécificité des images médiévales soulignée en général, il nous faut à présent distinguer la nature différente des images occidentales et byzantines. En effet, les images byzantines font l'objet de vénération particulière. Elles sont communément appelées icônes dans le sens large du terme englobant non uniquement les morceaux de bois peints, mais aussi les fresques, les ivoires, etc., et elles renferment la représentation du divin ${ }^{4}$. Un des premiers et plus célèbres théologiens de l'icône est sans doute Jean Damascène. Ces images particulières apparaissent comme des médiums entre le monde divin et celui des hommes, elles contiennent en elles une parcelle du prototype, et sont des manifestations visibles de la puissance invisible.

C'est dans un contexte de contestation et de destruction des images par des iconoclastes que naît la défense iconodoule des icônes en tant que théophanie et moyen de faire parvenir la vénération au modèle par l'image. Voici un passage du discours de l'un de ses plus fervent défenseur :

«qu'ils [ceux qui blâment les iconodoules] sachent qu'au commencement Dieu fit l'homme à son image... Devenu véritablement, essentiellement homme, il [le Christ] a passé sa vie sur terre, s'est mêlé aux hommes, a fait ses miracles, a souffert, a été crucifié, ressuscité et a monté au ciel. Toutes ces choses sont arrivées réellement, les hommes les ont vues, on les a reproduites en dessins pour nous en souvenir et pour l'enseignement de ceux qui n'y ont pas assisté en sorte que sans les avoir vues, mais en écoutant et en croyant, nous retrouvions la joie du Seigneur. Comme tout le monde ne savait pas lire ou n'en avait pas le loisir, les Pères ont vu dans ces icônes comme un bref rappel de ces actions sublimes. Souvent, alors même que l'on ne pense pas à la passion du Seigneur, en voyant l'icône de la Crucifixion du Christ, elle nous revient mémoire et, tombant à genoux, nous adorons celui qui est reproduit et non la matière; pas plus que nous n'adorons la matière des Evangiles ni la matière de la croix, mais l'image gravée dessus... La vénération pour elle rejaillit sur celui qui par elle s'est incarné. Pareillement les exploits de ces hommes saints nous poussent au courage, au zèle à imiter leur vertu et à chanter la gloire de Dieu. Comme nous le disons, la vénération pour la grandeur des serviteurs prouve nos bonnes pensées envers le commun Maître et la vénération de l'icône rejaillit sur le modèle. Il y a une tradition non écrite, comme d'adorer tourné vers l'Orient et beaucoup d'autres choses semblables; c'est elle qui nous fait adorer la croix... Les apôtres ont laissé bien des traditions non écrites...»."

4 Je ne voudrais pas m'arrêter ici sur les arguments du théologien qui, même s'ils mériteraient d'être discutés, sont par ailleurs bien connus comme l'usage didactique des icônes, leur fonction de mémoire, de support à la vénération, etc.

5 Et voici comment au VIIe concile de Nicée, en 787, le dogme s'affirme officiellement par un décret : 
« En effet, plus on les voit [l'image de notre Seigneur, Dieu et Sauveur Jésus-Christ, celle de notre Dame immaculée, la sainte Mère de Dieu, celle des anges, dignes de notre respect, celle de tous les saints et justes], grâce à leur représentation par l'image, plus en contemplant leurs images on est amené à se rappeler et à aimer les modèles originaux et à leur donner salutations et respectueuse vénération; non pas l'adoration véritable propre à notre foi, qui convient à la nature divine seule, mais comme on le fait pour la représentation de la glorieuse et vivifiante croix, pour les saints évangiles et tous les autres objets sacrés; ... l'honneur rendu à l'image s'en va au modèle original ${ }^{6}$.

6 Ainsi se met en place le statut particulier de l'icône porteuse de divinité. Et il en va de même non seulement pour les icônes acheiropoïètes, mais aussi pour celles faites de main d'homme. L'icône est considérée donc comme une réminiscence au sens de Platon, c'est-à-dire comme une restitution du prototype. L'image est là pour manifester Dieu et Dieu se manifeste dans et par l'image.

7 L'image rend présent le divin, mais qu'en est-il des personnages humains qui la peuplent? Par rapport à cette double spécificité des images byzantines que je viens de décrire, comment interpréter la présence figurative d'un personnage en train de prier ou d'offrir un don à la divinité dans l'icône même? Comment cet espace particulier où se côtoient le saint, le divin et l'humain fonctionne-t-il alors?

3. Prier, donner, asseoir son pouvoir en image

8 Les images byzantines ont la réputation, car elles-mêmes se veulent telles, d'être figées, reproduisant à l'infini un prototype. Or, nous assistons à une créativité importante dans la mise en place de schémas iconographiques et de combinaisons figuratives pour rendre un hommage individuel à la divinité. Dans le monde byzantin (élargi à l'Europe du Sud-Est), il existe de nombreuses occurrences de don personnel figuré dans l'image sur différents supports, à savoir - les fresques, les miniatures, les icônes (stricto sensu). Deux principaux critères servent ici à la réflexion : soit les images respectent une iconographie stable, soit elles inventent de nouveaux schémas pour intégrer un personnage historique contemporain ou non à l'histoire sainte/divine. Dans les deux cas, je chercherai les mécanismes de production de sens dans l'image en relation avec le texte (quand c'est possible). Il ne s'agit pas de catégoriser ou de classer les images, mais de s'interroger sur le lien entre l'image humaine et l'image divine qui partagent la même surface d'inscription. Plus que jamais la longue durée me semble intéressante pour observer ces phénomènes, des mosaïques de Justinien et Théodora à Ravenne, aux icônes valaques de la famille princière de Neagoe Basarab, en passant par les fresques serbes des rois Etienne. Je précise aussi que, malgré toute la richesse et la multitude des images en question, je m'arrêterai surtout sur la mise en place figurative du don dans une série de miniatures bulgares du XIVe siècle, comparés à leur modèle grec du XIe siècle, où le personnage historique, recevant un don ou les honneurs, fait irruption dans un schéma iconographique stable ou au contraire lorsqu'il sert de centre organisationnel dans une composition entièrement nouvelle, pensée pour lui. A titre de comparaison, j'évoque pour commencer d'autres manuscrits, plus tardifs, avec une présence princière, et qui font partie de la famille du manuscrit grec. Il s'agit notamment d'un manuscrit valaque de la fin du XVIe siècle (apporté en Moldavie au début du XVIIe siècle, offert au monastère de SuceviŢa, et connu comme le Suc. 23), et, l'un des deux manuscrits moldaves du XVIIe siècle'. Notons en ce qui concerne les manuscrits princiers Suc. 23 et Suc. 24 qu'il n'y a pas d'inscription concernant le don, à exception de celle sur la reliure du Suc. $24^{8}$. Elle est la suivante : 
«Ce tétraévangile a été commandé et il a été fait et relié en métal par le voïvode Ioan Ieremia Movilă et par sa femme la princesse (doamna) Elisabeta et par leur fils Constantin voïvode. Et il a été donné en leur mémoire au monastère qu'ils ont euxmêmes fait construire nouvellement, nommé SuceviŢa et qui est consacré à la fête de la Résurrection de notre Seigneur et Sauveur Jésus Christ, en l'an 7115 [1609], 25 mars ». (Suit le sceau du maître orfèvre avec les lettres GLI en cyrillique). et sa famille à l'église (qu'ils avaient fait construire). Par ailleurs, cet acte de bienfaisance est rendu éternel non seulement par le texte mais aussi par l'image (v. fig. $1 \mathrm{a}$ et $\mathrm{b})^{\circ}$. Dans ce but, le prince et sa famille sont représentés dans une miniature du manuscrit en question et sur une fresque de la dite église. Ils forment une procession devant le Christ trônant et ils lui rendent hommage en offrant soit le modèle de l'église (dans le cas de la fresque) soit un phylactère (dans le cas du manuscrit). Dans les deux cas, il s'agit d'une composition pensée pour le prince, et qui s'intègre dans un contexte iconographique propre au support d'inscription de l'image princière en question.

Les images du Suc. 23 présentent un autre prince et son fils. Quatre miniatures renferment la présence du prince Alexandru II et de son fils Mihnea en les mettant en contact avec les évangélistes : au fol. 97v - Alexandru, Mihnea et Mathieu; au fol. $152 \mathrm{v}$ Mihnea et Marc, au fol. 238v - Mihnea et Luc, et au fol. 303v - Alexandru et Jean (fig. 2a-d). Le Tétraévangile du prince (voïvode) Alexandru II date entre 1568 et 1577. Les personnages ne présentent ni ne reçoivent de dons. Ils font simplement des gestes de prière à la rencontre des évangélistes. C'est uniquement dans la dernière miniature que le prince tient une croix dans la main gauche et tend l'autre comme pour recevoir le manuscrit de l'évangéliste. La croix est à la fois le signe de la prière et l'expression de la foi. Si les images ne font pas référence explicite au don du prince ou de Dieu, ailleurs, on a pu directement mentionner le pouvoir comme donné par Dieu au prince. Ainsi, dans une inscription des panneaux votifs de BucovăŢ, où le prince est représenté avec sa famille, le pouvoir princier est mentionné en terme de don (v. fig. 3) (10 $^{10}$.

11 Ainsi donc, Dieu donne le pouvoir et en retour le souverain se doit, entre autres, de préserver, d'être le gardien la parole divine dans les manuscrits et sur les images. La présence du prince dans les images, aux côtés du Christ (et/ou de la Vierge) garantit la conservation de sa mémoire en tant que bienfaiteur. Des prières seront aussi adressées pour son âme afin que, le jour du Jugement dernier, il soit sauvé. Plus qu'une simple propagande, c'est donc d'abord un acte de foi de la part du souverain qui se remet au Sauveur. Le sens du don est produit par l'impact direct de l'image et l'écart entre les compositions iconographiques traditionnelles, et l'innovation et l'inventivité de cette intrusion dans le sacré.

Comme nous nous intéressons au lien entre texte et image dans la production du sens $\mathrm{du}$ don, nous allons remonter le temps pour nous préoccuper en particulier des inscriptions et des annotations qui accompagnent les images de don dans les manuscrits add. ms. 39627 et le ms. gr. 74, datant respectivement du XIVe et XIe siècles.

13 Arrêtons nous d'abord sur le Tétraévangile de Londres (ou de Curzon), qui se trouve à la British Library, sous la cote Add. MS. 39627, datant du XIVe siècle, produit par l'Ecole de Tarnovo, Bulgarie ${ }^{11}$. J'ouvre une parenthèse pour remarquer qu'au folio 5 , une annotation plus tardive mentionne que le manuscrit a été racheté par le prince moldave Alexandre le Bon au XVe s. L'inscription représente une prière pour le prince 
et elle demande que lui soit fait don de longévité. On voit ainsi le lien qui est fait entre la possession de la Bonne Parole et le salut de l'âme.

Mais revenons au texte et aux images du manuscrit du XIVe s. Les deux folios du frontispice (2v-3r) (fig. 4) renferment l'image de la famille du tsar Ioan Alexandre : sur le fol. $2 \mathrm{v}$ - le despote Constantin, gendre du tsar, et les trois filles du tsar, et sur le fol. $3 \mathrm{r}$ - le tsar, son épouse Théodora et deux de leurs enfants, dont l'héritier Ioan Šišman, portant le même costume que son père. On peut remarquer le détail intéressant du double geste de bénédiction par la droite divine. Ces deux images sont pensées indépendamment du programme d'ensemble du manuscrit et attestent une innovation de la pensée figurative, même si à Byzance de nombreux manuscrits portent l'image de leur commanditaire ou destinataire au début du manuscrit. Cependant, la composition de la scène est originale, inventée pour cette occasion. Il s'agit d'un hapax. Dans cette composition, le sens n'est pas induit par rapport aux autres images ou au texte, mais vient de la composition elle-même.

Si l'image novatrice ouvre le manuscrit, le texte, créé aussi pour l'occasion, le referme. En effet, le moine Simon a écrit l'éloge du tsar et l'a placé au colophon du manuscrit.

«Gloire à Dieu, célébré en la Trinité qui emplit de son être toute bonne action qui est entreprise en son nom et donne le commencement et la fin. Cette source de vie de la nouvelle grâce du très délectant enseignement du Christ et de ses témoins, disciples et apôtres, nommé tétraévangile, a été écrit non seulement, de l'extérieur, avec des couleurs et de l'or ou du lin tressé ou encore des gemmes et des perles, mais aussi, de l'intérieur, par l'effusion du verbe divin... Lorsqu'il eut cherché, le très chrétien, en Christ fidèle, le très grand et couronné de Dieu autocrate tsar Ioan Alexandre, il le trouva, comme une source de lumière, placé à un endroit sombre et oublié, resté inutilisé par les anciens tsars. Ce très chrétien tsar Ioan Alexandre l'a trouvé mu par une volonté divine et l'ayant montré, il [l'évangile] a été traduit du grec en notre langue slave et a été sorti au grand jour. Il a été enchâssé dans du bois doré, et à l'intérieur, il a été décoré artistement par des peintres avec des images vivifiantes de notre Seigneur et de ses disciples glorieux, dépeints avec des couleurs claires et de l'or. Ceci a été fait pour la confirmation de son règne. De même que le grand parmi les tsars tsar Constantin et sa mère Hélène a sorti des entrailles de la terre la croix vivifiante du Seigneur notre Dieu, ainsi celui-ci [Ioan Alexandre] a fait de même avec l'évangile. En ce temps, il tenait le sceptre de l'empire bulgare et grec avec sa très chrétienne, couronnée de Dieu et nouvellement convertie tsarine kyra Théodora, ce don de Dieu... et avec son très cher et bien-aimé fils le tsar Ioan Šišman; et pour la gloire du Créateur du monde et des évangélistes Matthieu, Marc, Luc, Jean, et par leurs prières qu'il reçoive de Dieu la victoire, la victoire sur les ennemis luttant contre lui et qu'il écrase leur tête sous ses pieds. Amen. Dans l'année 6864 de la création du monde, indiction IX. Et celui qui a écrit ce livre se nomme le moine Simon, serviteur de mon maitre, le tsar. ${ }^{12}$ "

Ce texte est intéressant en plusieurs points. Tout d'abord, il fait un rapprochement explicite entre l'invention de la croix par Constantin et Hélène et la découverte du manuscrit par le tsar. Il s'agit évidemment d'une action inspirée de Dieu et visant à le glorifier. Ensuite, il s'agit d'une action d'affirmation non seulement du pouvoir, mais aussi d'une identité face à Byzance - le manuscrit a été traduit du grec en slavon, souligne le texte. Le couronné de Dieu a reçu de lui le pouvoir, l'affirmation de son pouvoir - par la victoire sur les ennemis et par la succession sur le trône - le copiste glose le nom de la tsarine "don de Dieu». C'est de plus grâce aux prières des évangélistes que le tsar recevra la victoire sur ses ennemis de Dieu ${ }^{13}$. Ces échanges entre les deux mondes, qui ne sont pas du tout séparés en réalité dans la vision médiévale, se 
laissent cerner le mieux en termes de don et de contre-don: don de la couronne symbole du pouvoir, don de la victoire - affirmation sur le trône par Dieu au souverain, contre-don exprimé en actions pieuses comme la conservation de la parole divine dans l'évangile de la part du souverain.

D'autres miniatures incluent l'image du tsar, mais elles font preuve de moins d'inventivité, car elles suivent une iconographie déjà existante. Le personnage du tsar fait une irruption au sein de compositions plus ou moins fixes. Ainsi donc, toutes les autres enluminures avec l'image du tsar sont peintes d'après le modèle d'un manuscrit grec du XIe siècle qui se trouve actuellement à la BnF, sous la cote ms. gr. $74^{14}$. Originellement, dans le manuscrit du XIe siècle, chaque évangile se termine par une enluminure où le destinataire et/ou commanditaire du manuscrit reçoit de l'évangéliste respectif en don soit un livre, soit un bâton, soit une autre marque de respect. Le personnage historique porte des habits de moine et son titre est celui d'higoumène, le supérieur hiérarchique d'un monastère orthodoxe. Son identité réelle est incertaine, mais on sait que le manuscrit porte un feuillet placé à la fin, qui contient une épigramme s'adressant à un empereur ${ }^{15}$.

Mise à part l'image incluse dans le manuscrit, et sur laquelle je reviendrai un peu plus loin, une autre particularité du manuscrit grec est que chacun des quatre évangiles se termine par une épigramme écrite en onciales (en majuscules) (fol. 62, 102, 165, 213-123v) qui glose précisément l'image de l'un des évangélistes et de l'higoumène. Voici les textes respectifs:

\section{$\mathrm{Au}$ fol. 62 de l'évangile de Matthieu}

«Puisque le premier et grand Pasteur qui, après avoir sauvé en toute Sagesse la brebis égarée, après l'avoir excellemment embellie par ses manières pastorales, $t$ 'a fait chef de ce troupeau vraiment honorable du Prodome (Jean-Baptiste), il convenait tout à fait qu'avec des paroles vivifiantes tu nourrisses tes sujets comme en des prés d'herbe. Accepte donc ce livre divin auprès de quoi rien parmi toutes choses n'est meilleur et, rassasiant ceux-ci (tes sujets) avec un juste discernement, nourris-toi toi-même en tout temps jusqu'à satiété ».

Au fol. 102 de l'évangile de Marc

«L'apôtre dit : Christ, vois-tu avec quelle attitude ton suppliant et adorateur s'approche de toi?

Le Christ répond : C'est avec une attitude lamentable et fort tendue, mais dis-moi quelle demande il apporte, car celui-ci ne profère aucune parole.

L'apôtre : Verbe de Dieu, celui-ci, se servant de moi comme intermédiaire, a jugé bon par prudence de se taire; il demande de recevoir le salut de son âme et la grâce d'une protection parfaite afin qu'après avoir dirigé maintenant cette vénérable institution, il reçoive la récompense des maîtres sages.

Le Christ : Marc, il ne manquera pas d'obtenir ce qu'il demande. »

21 Au fol. 165 de l'évangile de Luc (la miniature a disparu dans le manuscrit grec)

" Le divin Luc a décrit en toute beauté les particularités de cette hiérarchie conforme à la loi dont Zacharie a revêtu la grâce. Car il lui fallait, lui qui avait été instruit dans les lettres et s'était excellemment exercé à toute sorte d'écriture, ne laisser tomber aucun des détails strictement requis, mais par sa justesse de langage et par l'envergure de ses pensées présenter le récit le plus somptueux. C'est assurément selon toute convenance que le peintre présente le chef du monastère debout et en train de recevoir de ses mains sacrées le signe distinctif du costume sacerdotal."

Au fol. 213-213v de l'évangile de Jean 
«Ce n'est pas de Sion, Père, mais du ciel qu'arrive cet homme inspiré aimé du Christ apportant le bâton don de Dieu. Accepte ce don, cachet de préséance ou, pour mieux dire, bâton de la puissance divine. Etant le maître au milieu de tous les ennemis, chante avec empressement, comme écrit le prophète inspiré de l'Esprit, la louange de l'auteur du bien. " 
l'higoumène (fol. 61v). L'architecture sommaire fait allusion à un édifice religieux surtout par les croix et les cierges.

Par contre, l'image du manuscrit bulgare suit la composition de l'image grecque en la simplifiant. Le tsar ne reçoit pas de livre, mais une inspiration divine (fol. 88r.) (fig. 5), alors que dans le manuscrit valaque, le prince et son fils présentent leur prière à l'évangéliste (fig. 2a).

Plus loin, à la fin de l'évangile de Marc, l'higoumène et le tsar reçoivent respectivement une bénédiction de la droite divine. Cette nouvelle scène, pensée pour le manuscrit grec, est incluse au-dessous de la scène de l'Ascension du Christ à laquelle assistent les apôtres, un ange et la Vierge et saint Jean en prière. (fol. 101 v. du ms. gr., et fol. 134v du ms. slav.) (fig. 6). Dans le manuscrit valaque, c'est Mihnea qui prie Marc, et tous les deux sont figurés dans une architecture conventionnelle séparément (fig. 2b).

Comme on l'a déjà noté, le folio avec la miniature de l'higoumène et de saint Luc manque dans le manuscrit grec (fol. 164v.). Pourtant, d'après les traces sur le feuillet 165 , on a pu établir que tout en haut se trouvait une image de la Pentecôte, et probablement au-dessous était figurée la rencontre entre l'higoumène et saint Luc ${ }^{18}$. Seulement puisque le manuscrit bulgare ne reprend pas la composition, on a conclu que lors de sa traduction et de son enluminure, le feuillet était déjà perdu. L'enlumineur s'est ainsi vu dans l'obligation d'innover et de trouver une composition originale. Cette dernière situe la rencontre entre le tsar et l'évangéliste tout en haut du folio (fol. 212v, fig. 7). Entre les colonnes, on a représenté les emblèmes du pouvoir royal et du sacerdoce : une lance avec le bouclier et une épée du côté du tsar, et, du côté de l'évangéliste, l'autel, l'évangile, l'étole. En-dessous de la miniature sont annoncés les chapitres de l'évangile suivant. Dans le Suc. 23, c'est Mihnea qui remplace le tsar dans la même composition - entouré de la lance avec le bouclier et de l'épée. Il n'y a pas d'autre texte en dessous de la miniature (fig. 2c).

31 Le dernier épisode de ces rencontres surnaturelles se clôt par l'entrevue de l'higoumène et de saint Jean (au fol. 213). Dans le registre supérieur apparaissent les apôtres, alors que dans le registre inférieur, sous les arcs d'une composition architecturale simplifiée et jouxtée par des arbres l'higoumène reçoit en même temps le bâton de l'évangéliste et la bénédiction de la droite divine. En dessous de la miniature commence déjà le poème traduit plus haut.

Dans la miniature bulgare, on a copié les apôtres et en dessous le tsar reçoit l'évangile des mains de saint Jean (fol. 272v., fig. 8). Dans le Suc. 23, c'est le prince Alexandru qui, une croix dans la main gauche, tend la droite en direction de saint Jean comme pour recevoir le manuscrit (2d).

On pourrait chercher des combinatoires sémantiques entre la présence du personnage et de l'évangéliste en dessous des scènes comme l'envoi des apôtres en mission, l'Ascension (et éventuellement la Pentecôte). Leur présence au sein même de l'historia (à la fois l'histoire sainte et l'agencement narratif des images) me semble déjà pleinement significative.

34 L'articulation d'images stables, codifiées et des images novatrices pensées pour l'occasion donne à voir comment l'imagerie byzantine s'accommode des modèles qu'elle est supposée suivre. Elle permet aussi de suivre dans la longue durée l'évolution des compositions iconographiques. Dans une tradition qui se veut conservatrice, respectueuse du modèle, le moindre écart prend sens. 

travers les siècles est l'intrusion d'un personnage au sein même de l'espace sacré. Jusqu'à présent, le personnage apparaissait dans des images pensées pour lui. Mais dans la dernière miniature que je vais présenter, il prend place au sein d'une composition déjà existante. Et cette intrusion fait sens pour lui (et aussi pour nous, spectateurs), mais sans qu'elle vienne à modifier la signification d'ensemble de la scène. Il s'agit de la miniature du Jugement dernier. (fig. 9). Elle est disposée au folio 93v du manuscrit grec, et au folio $124 \mathrm{du}$ manuscrit bulgare. Dans le manuscrit grec, il s'agit de l'higoumène (mais son image se dédouble, lui - nimbé, tourné vers la Vierge, et une communauté de moines, tournée vers Abraham), dans le manuscrit bulgare - il s'agit uniquement $\mathrm{du}$ tsar. L'intention est assez claire. Par cette image, le personnage souhaite obtenir, sinon réserver, sa place au Paradis. Il se place (ou est placé) parmi les Justes, et présente sa prière à la Vierge orante. La particularité du manuscrit grec est que devant Abraham se tient une autre figure, qui semble résumer la communauté monacale entière, et donc fait en sorte que la prière pour le salut de l'âme a un caractère moins individuel que celle du tsar bulgare. On peut aussi souligner que dans le manuscrit valaque, les princes ne sont pas représentés dans cette scène du Jugement dernier. Sans doute, cette absence témoigne-t-elle d'une réception différente de l'audace de leurs « prédécesseurs ».

analysons cette présence dans les manuscrits bulgare et grec. Comme on l'a noté plus haut, la particularité de l'image byzantine c'est qu'elle projette en même temps l'image céleste sur terre et fait communiquer par la vénération qui lui est rendue les hommes et le divin, c'est aussi qu'elle re-présente (rend présent) les personnages dans l'historia et les acteurs de cette histoire sainte dans le monde des hommes. Elle se veut être un médium entre les deux mondes. Ce statut particulier de l'image byzantine donne sens à la présence humaine dans l'espace sacré, et à l'expression du désir de salut par l'image.

En fonction des différents niveaux de lecture qu'on peut pratiquer (le manuscrit dans sa matérialité, la terminologie utilisée, la temporalité dans les images et dans le texte), je voudrais attirer l'attention sur plusieurs points qui me semblent importants pour mon propos: tout d'abord l'opposition qui est faite dans le texte du colophon du manuscrit bulgare entre les images, l'ornementation, les matériaux de fabrication du manuscrit qui sont mentionnés comme une écriture extérieure, et le Verbe, la parole divine, qui elle est "intérieure ", et qui s'est incarnée, a pris chair dans l'écriture. Ensuite, en rapport avec le don, dans la terminologie du manuscrit grec, le pouvoir impérial (et son emblème - la couronne), le pouvoir religieux (et ses emblèmes - le bâton etc.), mais aussi le salut, sont bien mentionnés en terme de don. Et enfin, aussi bien dans le texte que dans les images des manuscrits, il est intéressant de relever le télescopage des temporalités - celle de l'humain et celle du divin, celle de la memoria et celle de l'historia, celle du présent et celle de l'avenir - du jour du Jugement dernier. Dans cette temporalité complexe l'homme se projette devant Dieu, mais Dieu est aussi présent dans l'immédiat, au moment même du message qui lui est adressé à la fois à travers le Verbe et à travers l'image.

38 Le message sotériologique est contenu dans ces images eschatologiques. L'espoir de salut par la prière et les bonnes œuvres se matérialise de façon plus ou moins autonome dans l'image. 
39 Ainsi, à partir du IXe siècle, avec la reconnaissance de la sacralité recelée dans l'icône et l'acquisition d'un statut officiel des images dans l'empire, tout un système de pensées figuratives se met en place et l'action d'élection divine et celle de générosité impériale peuvent désormais se rencontrer dans l'image porteuse de salut ${ }^{19}$. Mais si l'évergétisme a pu, dans certains cas, être détourné vers le rachat des péchés, tel n'était pas son sens initial. Le don impérial, y compris à travers l'aide pour la fabrication de manuscrits, se voulait être un hommage à celui qui l'avait élu et avait placé dans ses mains le bon gouvernement de l'empire. Seulement, même le meilleur des souverains ne pourra jamais rendre autant, voire plus, qu'il n'a reçu et ce fait le condamne à rester toujours dans une position d'infériorité par rapport à Dieu qui est le donateur universel et premier.

4. Le sens du don chrétien

40 Il est nécessaire de replacer la problématique du don, du pouvoir et de la foi, dans leur contexte théologico-historique. Parmi les fidèles, l'empereur est à Byzance, et dans le monde byzantin, le plus haut placé dans la hiérarchie sociale. Il faudrait s'adonner à un véritable exercice de reconstitution de la mentalité médiévale de l'époque à travers une mosaïque de textes et d'images, car il n'existe pas vraiment de théorie du don. Les textes théologiques des Pères de l'Eglise sur le don et le sacrifice nous donnent des indices pour penser le don. Je ne mentionnerai que ceux d'Eusèbe de Césarée ${ }^{20}$, saint Augustin ${ }^{21}$, Maxime le Confesseur. ${ }^{22}$ Les écrits des trois exégètes sur le sacrifice vont dans le même sens. Sans que ce soit explicité, on peut tout de même percevoir une inversion dans la logique du don. En effet, pour présenter un peu schématiquement la pensée théologique chrétienne, depuis l'avènement du christianisme, le sens du don a changé en s'inversant. Auparavant, dans le paganisme et encore avec le judaïsme, on pouvait donner à Dieu pour l'apaiser, en obtenir des faveurs, essayer de se racheter. Avec le christianisme, c'est Dieu qui devient le donateur premier. Il offre son Fils pour le rachat du péché originel de l'homme. Le Christ donne sa vie pour le salut de l'homme. Les holocaustes et les sacrifices ne sont plus nécessaires de la même manière qu'ils ne l'étaient avant cette ère. Ainsi, tout bienfait sur Terre vient de son Créateur qui en devient le donateur premier. Pour se sauver l'homme doit imiter le Christ philanthrope et évergète, et s'engager dans ce rapport d'échange, mais dans lequel il ne pourra tenir que le second rôle et comme il ne pourra jamais donner autant qu'il a reçu, il restera dans une position de subordination et d'infériorité par rapport à Dieu.

41 Les textes liturgiques comme la Divine liturgie de saint Jean Chrysostome témoignent de leur côté aussi de la manière de donner pour le fidèle :

"Je viens à Toi inclinant la tête et je Te supplie, ne détourne pas de moi ta Face et ne me rejette pas du nombre de tes enfants, mais rends-moi digne, tout pêcheur et indigne serviteur que je suis, de T'offrir ces dons. Car c'est Toi qui offres et qui es offert, Toi qui reçois et qui es distribué, ô Christ notre Dieu... »"

42 Mais le don se retrouve à plusieurs niveaux dans les échanges socio-religieux. A part le don « existentiel » fait à l'homme en général, Dieu (le Christ) peut récompenser son fidèle serviteur par des dons concrets, et notamment par le don du pouvoir. Comme il est bien connu, dans le monde byzantin, c'est le souverain qui se veut le garant de la foi. C'est la raison pour laquelle il a reçu le pouvoir qui doit être exercé au nom de Dieu. Et l'assimilation des premiers empereurs à des apôtres est bien connue, comme notamment dans le cas de Constantin et de sa mission décrite par Eusèbe ${ }^{24}$. On peut faire correspondre ses écrits à la description de la mission des apôtres: 
«Dieu donnait son pouvoir...à qui Il voulait... Il les avait appelés sans que rien en apparence ne justifiât son choix. Il leur avait donné son amour et les avait chargés d'une mission... " ${ }^{25}$. don justement à la lumière de l'hommage dû à Dieu, de l'obligation de rendre ce qui est reçu et de penser à la mort ${ }^{26}$. Sainte Euphrosynè inspire à l'empereur plusieurs règles de comportement. Le geste de commanditer des manuscrits pourrait, donc, être interprété non seulement comme un engagement à étudier les Saintes Ecritures selon les conseils de la sainte, mais aussi de contribuer à leur diffusion et connaissance, et celui de se représenter dans un contexte funéraire et eschatologique - comme la réponse à sa recommandation de craindre Dieu et de toujours penser à la mort. Ainsi, le sens habituellement rattaché au don comme "don pour le salut de l'âme", pourrait renvoyer précisément à cette inquiétude perpétuelle de la mort comme le prêchait explicitement le discours mentionné. Ce texte est un témoignage précieux sur la mentalité de l'époque, sur le rapport que devait entretenir le souverain avec l'au-delà, et il permet de revenir au sens premier de l'échange.

Il me semble aussi que l'usage politique des images, s'il fallait séparer le politique et le religieux, n'est que secondaire même dans le cas du souverain ${ }^{27}$. Supposer que le souverain (ou son entourage) pouvait essayer d'asseoir son pouvoir à travers les images paraît exagéré. Le souverain avait une légitimité qu'aucune image ne pouvait garantir. Par contre, l'image pouvait donner à voir les rapports particuliers entre le souverain et Dieu dans la quête du pouvoir sur terre et du salut dans l'au-delà. Dans ces rapports surnaturels, le don joue un rôle tout à fait central et fondamental. Nous venons donc d'analyser le sens global du don chrétien et ses actualisations à travers des images concrètes dans la longue durée. Qu'elles soient innovantes ou au contraire moins inventives, les images sont toutes porteuses d'un espoir de salut, notamment à travers le don. Formellement cet espoir s'exprime de différentes manières, mais finalement les faisceaux sémantiques se rejoignent au fond: au don premier de Dieu correspond une série d'actes qui engagent le destinataire de ce don premier. Les représentations et les inscriptions donnent à voir à leur manière ces rapports de don et de contre-don. L'être humain - et à plus forte raison le souverain, côtoie le divin ou le saint dans un espace très particulier qui tient à la fois de l'imago et de l'eikôn.

\section{NOTES}

1. Texte à paraître dans la Revue des Etudes Sud-est Européennes, Bucarest, 2008. 
2. V. J.-Cl. Schmitt, « Anthropologie Historique », communication, Buenos Aires, octobre 2006 ; idem, "L'historien et les images », in Der Blick auf die Bilder, Gottingen, p. 9-51; ibidem, Le corps des images. Essais sur la culture visuelle au Moyen Age, Paris, Gallimard, 2002 ; J. Le Goff et J.-Cl. Schmitt., "L'histoire médiévale », in Cahiers de civilisation médiévale Xe-XIIe siècles, Centre d'études supérieures de civilisation médiévale, Poitiers, 1996, p. 9-25; H. Belting, Image et culte. Une histoire de l'art avant l'époque de l'art, Paris, Le Cerf, (pour la trad. fr.) 1998; A. Burguière, L'école des annales. Une histoire intellectuelle, Odile Jacob, Paris, 2006.

3. Cf. J.-Cl. Schmitt, «La culture de l'imago ", «Images médiévales », Annales, An. 51-n¹, 1996, p. 3-36 ; idem, «Images », dans Dictionnaire raisonné de l'Occident médiéval, (dir.) J. Le Goff et J.-Cl. Schmitt, Paris, Fayard, 1999, p. 497-511.

4. Sur la différence entre image et icône, v. A. Vasiliu, «Voir, nommer, regarder une image à la fin de l'Antiquité ", Miltos Garidês (1926-1996) afierôma, Panepistêmio Iôanninôn, Tomeas arhaiologias, Iôannina, 2003, p. 813-845, p. 817.

5. J. Damascène, La foi orthodoxe, suivie de Défense des Icônes, préface de J. Kovalevsky, traduction, introduction et notes docteur E. Ponsoye, publication de l'Institut orthodoxe français de théologie de Paris, Saint-Denys, diffusion Editions Cahiers StIrénée, Paris, 1966, p. 184 et s.

6. G. Alberigo, Les conciles œecuméniques, Les décrets, tome II-1, Paris, Le Cerf, 1994. Un second iconoclasme est déclenché en 815 jusqu'au 11 mars 843 .

7. Sur ces manuscrits, v. entre autres, Sirarpie Der Nersessian, "Two Slavonic Parallels of the Greek Tetraevangelia: Paris. gr. 74”, The Art Bulletin, vol. IX, 1927, 3, p. 222-274 ; G. Popescu-Vilcea, Un manuscris al voievodului Alexandru al II-lea, Bucurest, Editura Meridiane, 1984 ; idem, Un manuscris al voievodului Ieremia Movila , Bucuresti, 1984 ; C. Costea, « Une nouvelle réplique slavonne du Paris. Gr. 74 : seven decades after », Revue Roumaine d'Histoire de l'Art. Série Beaux-Arts, Bucarest, Editura Academiei Române, 2003, Vol. 38 (2001), p. 3-17 (v. sur Internet : http://archaeology.kiev.ua/journal/030501/ costea.htm).

8. Je remercie tout particulièrement ConstanŢa Costea pour les renseignements et les images qu'elle a pu me fournir concernant ces sources roumaines.

9. Pour la parenté sémantique des images de don dans le manuscrit et sur la fresque de SuceviŢa, v. fig. 1.

10. Cf. fig. 3 pour les images; et pour le texte de la dite « chronique de BucovăŢT, v. I. Bogdan, Cronicile slavo-române din secolele XV-XVI, édition revue par P. Panaitescu, Bucarest, Editura Academiei Republicii Populare Romîne, 1959, p. 194-196 : «domnul dumnezeu m-a dăruit cu domnia » (trad. en roumain), p. 195.

11. Sur ce manuscrit, il existe un grand nombre d'études. E. Bakalova, « La société et l'art en Bulgarie au XIVe siècle ", Actes du XIVe Congrès international des études byzantines, Bucarest, vol. II, 1971-1975, p. 32-38 ; idem, « Ivanovskite stenopisi i ideite na isikhazma ", Izkustvo, Sofia, 1976, 9, 14-21; ibidem, « Ktitorskite portreti na car Ioan Aleksandâr kato izraz na politiceskata i religioznata ideologia na epokhata », Problemi na izkustvoto, Institut za izkustvoznanie de BAN, Sofia, 4, 1985, p. 45-57 ; T. Velmans, « Le portrait dans l'art des Paléologues ", " Art et société à Byzance sous les Paléologues ", Actes du Colloque organisé par l'association internationale des Etudes byzantines à Venise en sept. 1968, Venise, 1971, p. 93-148 ; L. Živkova, Cetveroevangelieto na Ivan Alexandar, Sofia, Nauka i izkustvo, 1980, E. Dimitrova, The Gospels of Tsar Ivan Alexander, Londres, The British Library, (1989) 1994 ; T. Kambourova, « Image et hiérarchie dans une miniature bulgare du XIVe siècle ", in Cahiers balkaniques, (dir.) T. Velmans, n 31, Paris, 2001, p. 139-147. 
12. La traduction est de moi.

13. Le don de la gloire sur les ennemis et de la longévité du pouvoir est mentionné aussi dans le Psautier de Sofia du tsar Ioan Alexandre. Pour le texte, v. E. Bakalova, « Portretat na tsar Ivan Alexandar ot Sofijskia pesnivetz : « realisam » ili kompilatzia ot topossi ? ", in Slovensko srednovekovno nasledje, Belgrade, 2001, p. 45-58.

14. Sur ce manuscrit, voir, entre autres, $\mathrm{H}$. Omont, Fac-similés des manuscrits grecs datés de la Bibliothèque nationale du Ixe au XIVe siècle, Paris, 1881 ; idem, Evangiles avec peintures byzantines du XIe siècle. Reproduction des 361 miniatures du manuscrit grec 74 de la Bibliothèque nationale, Paris, 1908 ; S. Der Nersessian, « Recherches sur les miniatures de Parisianus graecus 74 », JOB, 21, Festschrift fur Otto Demes zum 70, Geburtstag, Vienne, 1972, p. 109-119 ; J.-G. Violette, « Etude des miniatures du manuscrit grec 74 », (thèse), Paris, 1976.

15. On peut supposer qu'il s'agit d'Isaac Ier Comnène (1057-1059), qui avait fait des dons au monastère et qui peut être vu comme le destinataire de ce manuscrit enluminé. Il se réfugie au Stoudios pour y finir sa vie. Ce manuscrit a été récemment attribué au copiste Théodore de Césarée.

16. Cf. J.-G. Violette, «Etude des miniatures...», p. 25. Je remercie Elka Bakalova d'avoir attiré mon attention sur le texte.

17. Violette souligne que pour des raisons codicologiques, il conclue que le folio où est inscrite la prière pour l'empereur n'appartenait pas au manuscrit d'origine, mais qu'il n'est pas impossible qu'il ait été rajouté à l'ensemble (à la même époque), idem, p. 37. Par ailleurs, on sait que Isaac Ier Comnène se retire du pouvoir, prend l'habit et meurt dans ce monastère. Le manuscrit a pu lui appartenir.

18. Violette, ibidem, p. 35.

19. Auparavant, Grabar recense trois cas où l'empereur et le Christ partagent le même espace, mais sans qu'il y ait une communication entre eux.

20. P. Maraval, Eusèbe de Césarée. La théologie politique de l'Empire chrétien. Louanges de Constantin, Paris, Cerf, 2001, p. 90 et s. : «Ce très grand sacrifice, le roi le célèbre pour tous, mais il sacrifie comme un bon pasteur : non en « immolant de splendides hécatombes d'agneaux premiers-nés ", mais en conduisant les âmes du troupeau raisonnable qu'il fait paître à la connaissance (de Dieu) et à la piété. » Et plus loin, il explique que les offrandes sont des actions de grâce dédiées au Protecteur et Sauveur. C'est Dieu qui donne la victoire, la paix, le salut des âmes et le souverain n'a qu'à répondre à ses dons par des actes pieux, comme la construction d'églises. «Car seul convient à Dieu ce sacrifice que le roi de notre pays a appris à célébrer avec des pensées purifiées, sans feu ni sang, en affermissant sa piété par des doctrines qui ne trompent pas son âme, en proclamant la doctrine de Dieu par un discours admirable, en cherchant à imiter la philanthropie du Tout-Puissant par des actions royales, en se vouant tout entier à lui et en s'offrant lui-même, prémices du monde qui lui a été confié, comme un grand don. » (p. 91)

21. V. la Cité de Dieu, ch. V et VI : «Ainsi le vrai sacrifice, c'est toute œuvre accomplie pour s'unir à Dieu d'une sainte union, c'est-à-dire toute œuvre qui se rapporte à cette fin suprême et unique où est le bonheur. C'est pourquoi la miséricorde même envers le prochain n'est pas un sacrifice, si on ne l'exerce en vue de Dieu. Le sacrifice en effet, bien qu'offert par l'homme, est chose divine, comme l'indique le mot lui-même, qui signifie action sacrée. Aussi l'homme même consacré et voué à Dieu est un sacrifice, en tant qu'il meurt au monde pour vivre en Dieu; car cette consécration fait partie de la miséricorde que chacun exerce envers soi-même » (ch. VI). V. aussi Le sacrifice dans les 
religions, colloque organisé sous la direction de Marcel Neusch, Beauchesne, Paris, 1994, et plus particulièrement le développement de $\mathrm{M}$. Neusch, «Une conception chrétienne du sacrifice. Le modèle de saint Augustin ", ch. V, p. 117-138, et surtout p. 123, où l'auteur insiste sur une inversion qui se produit avec le christianisme, explicitée par saint Augustin, à la suite de laquelle tout don vient de Dieu et le contre-don consiste en une réponse de l'homme, qui n'est pas sacrificielle, mais existentielle.

22. « La preuve claire de la grâce de participer au sort des saints dans la lumière... est la disposition volontaire de bienveillance envers le semblable. L'œuvre de celle-ci est que l'homme qui en quelque manière a besoin de notre aide, nous devienne en puissance familier comme Dieu et qu'il ne reste pas sans soin ni providence, mais que par l'ardeur appropriée soit montrée en acte et vivante notre disposition envers Dieu et envers le prochain. Car l'œuvre est la preuve de la disposition. En effet, rien n'est aussi facile pour la justification, ni convenable pour la divinisation et la proximité avec Dieu, que la miséricorde de l'âme offerte avec plaisir et joie à ceux qui en ont besoin. Car si le Verbe a montré que celui qui a besoin de bienfaisance est Dieu - car, dit-il, « Ce que vous avez fait à l'un de ces plus petits, c'est à moi que vous l'avez fait » (Mt 25/41) - Dieu qui l'a dit montrera d'autant plus qu'est vraiment Dieu par grâce et participation celui qui pouvait faire du bien et l'a fait parce qu'il a pris par une bonne imitation l'énergie et la propriété de sa propre bienfaisance... ». Cf. A. Riou, Le monde et l'église selon Maxime le Confesseur, Paris, Beauchesne, 1973, p. 169.

23. La Divine liturgie de Saint Jean Chrysostome, Paris, Liturgica, 1999, p. 37.

24. Op. cit, ch. III, p. 92 et s. : « Et celui-ci, qui se réjouit d'une telle offrande et reçoit volontiers ce don, satisfait du hiérophante de ce splendide et magnifique sacrifice, ajoute à son règne de nombreuses années de plus, accroissant ses bienfaits envers lui pour le récompenser des cérémonies en son honneur. Il lui accorde, avec beaucoup de bienveillance, de célébrer les multiples fêtes de la monarchie en désignant à chaque retour de la panégyrie décennale un de ses fils comme associé du trône royal et en lui accordant une prolongation de temps, comme à une plante florissante et dans toute sa force. » V. aussi ch. V, p. 101 : « ... lui [l'empereur] qui a été orné par Dieu de vertus innées et a reçu dans son âme les émanations d'en haut. Il est devenu raisonnable de par la raison universelle, sage par participation à la sagesse, bon par la communion de la bonté, juste parce qu'il participe à la justice, tempérant grâce à l'idée de tempérance, courageux en ayant part à la force d'en haut. »

25. C'est ainsi que J.-P. Roux présente la vocation des apôtres et disciples du Christ. Un parallélisme entre les apôtres et les souverains a été mis en évidence par les premiers théoriciens du pouvoir impérial chrétien et, notamment, par Eusèbe de Césarée. (Pour la citation, v. J.-P. Roux, Le roi. Mythes et symboles, Paris, Fayard, 1995, p. 246).

26. Cf. C. A. Bourdara, « Le modèle du bon souverain à l'époque de Léon VI le Sage et la vie de sainte Euphrosynè », EYYYXIA. Mélanges offerts à Hélène Ahrweiler, t. 1, Centre de recherches d'histoire et de civilisation byzantines, Paris, Publications de la Sorbonne, 1998, p.109-117. Voici une citation de certains des conseils : «1) rendre à Dieu des honneurs inégalables. La raison spéciale en est que l'Empereur est supérieur en honneurs à tous les autres hommes. Il est donc obligé de rendre les mêmes honneurs à celui qui l'a honoré ; 2) employer l'autorité légalement... ; 3) rendre à l'Eglise la part d'honneur qui lui revient ; 4) se comporter envers ses sujets comme envers des coserviteurs et comme un père ; 7) être prompt à la bienfaisance ; 14) étudier continuellement les Ecritures Saintes, quand il n'a pas d'occupations séculières ; 16) craindre Dieu et obéir à ses ordres ; 17) penser continuellement à la mort et en 
parler... Il doit se rappeler que ce n'est pas la puissance qui sauve l'Empereur, mais seulement les armes de la justice. C'est ainsi qu'il sera proche de Dieu. » (p. 113 et s.). 27. On sait, par ailleurs, depuis les travaux plus récents des historiens comme J. Le Goff qu'il ne faut guère séparer le politique et le religieux à cette époque, car les deux aspects sont liés.

\section{RÉSUMÉS}

Les images médiévales ont un statut particulier. Ceci est d'autant plus vrai pour les images byzantines, et plus particulièrement pour les icônes. Mais comment interpréter la présence de personnages contemporains à la production des miniatures et des fresques au sein même d'un tel espace sacré, destiné à présenter l'histoire sainte/divine? Souvent, le souverain - seul ou en famille, offre ou reçoit un don dans les images. Nous analyserons les occurrences textuelles et figuratives du don impérial/princier et/ou du don surnaturel destiné au souverain dans quatre manuscrits de la même famille : le ms. gr. 74 (Paris, Bibliothèque nationale de France), le add. ms. 39627 (Londres, British Library), le suc. 23 et le suc. 24 (Sucevita, Roumanie), pour terminer par une interrogation plus globale sur le sens du don.

\section{INDEX}

Mots-clés : don, image byzantine

Index géographique : Byzance

\section{AUTEUR}

\section{TANIA KAMBOUROVA}

Groupe d'Anthropologie historique de l'Occident médiéval (EHESS-CNRS, Paris) 